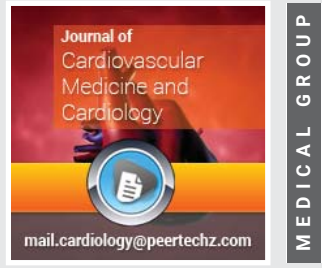

\title{
Esophageal temperature evolution during high power short duration ablation: Numerical simulations and clinical suggestions
}

Received: 05 May, 2020

Accepted: 14 May, 2020

Published: 15 May, 2020

*Corresponding author: Antonio Fasano, Professor, FIAB, R\&D, University of Florence, SpA, via Passerini 250039 Vicchio (Firenze) Italy, Tel: +39 3440227030; Email:a.fasano@fiab.it

Keywords: Atrial fibrillation; High power short duration ablation; Radiofrequency; Esophageal temperature; Esophageal thermal lesionsc

https://www.peertechz.com

\section{Check for updates}

\author{
Antonio Fasano ${ }^{1,2 *}$ and Luca Anfuso ${ }^{1}$ \\ ${ }^{1} \mathrm{FIAB}$, Florence, Italy \\ ${ }^{2}$ Department of Mathematics and Informatics U. Dini, Univ. of Florence, Italy
}

\section{Introduction}

In recent times electrophysiologists started considering the possibility of performing Radiofrequency (RF) ablation for the treatment of atrial fibrillation by delivering shorter pulses of larger power (e.g. $60 \mathrm{~W}, 6 \mathrm{~s}$ applications). Such a technique is known as High Power Short Duratio (HPSD) Ablation [1-5].

The key point of HPSD ablation is that it allows getting results quite comparable to the traditional approach in shorter time, thus reducing patients' discomfort and fluoroscopy time. Some electrophysiologists [6], are even testing very HPSD (90W, $4 \mathrm{~s}$ applications). It is well known that esophageal thermal lesions are among the most feared complications. In this respect, it has been pointed out that HPSD ablation may force Luminal Esophageal Temperature (LET) to reach dangerous values [7]. Here we want to exploit a mathematical model introduced in a previous paper about traditional RF ablation in order to investigate the thermal effects of a HPSD procedure on the esophagus. More precisely, we address the following questions:

Is HSPD safe and is there a preferable sequence when ablating around the Pulmonary Vein (PV) closest to the esophagus?

How sensitive is LET increase to apparently unimportant differences in the patient's anatomical configuration? This aspect can be of great importance considering that the tissues compression induced by exerting a contact force on the ablator may reduce the esophagus-ablator distance to some extent.

\section{Methods}

In several papers [8-12], mathematical models have been used to investigate the thermal field around the ablator, both for $\mathrm{RF}$ and cryoablation procedures.

Here we adopt the model by Fasano, et al. [11]. The equations to be integrated are the so-called bioheat equation, describing the evolution of the thermal field with a source term due to $\mathrm{RF}$ power absorption, $\rho c \frac{\partial T}{\partial t}-k \Delta T=\rho_{b} c_{b} \omega\left(T_{b}-T\right)+Q, \quad(T$ temperature, $\rho$ density, $c$ specific heat, $k$ thermal conductivity, $Q$ heat supply rate p.u. volume, $\omega$ blood perfusion rate, index $b$ refers to blood, not indexed symbols refer to tissues), and the equation for the electric potential $V, \Delta V=0$, (in both equations $\Delta$ is the Laplace operator). The heat source $Q$ due to $R F$ power absorption (metabolic heat is neglected) is given by $Q=\frac{1}{2} \sigma E^{2}$ where $\sigma$ is the electric conductivity and $E=|\nabla V|$ is the electric field (the factor $1 / 2$ accounts for the fact that the potential is computed as a static quantity while the RF field is oscillating).

The equations above are solved in a domain representing the patient's thorax, sketching the heart as a fixed domain (heart beats have little influence on the thermal field), as shown in Figure 1. keeping into account the medium inhomogeneity. For more details on the model and for the values used for the various thermal and electric parameters the reader is referred to quoted paper. 
An irrigated ablator is applied at 6 evenly spaced points around the ostium of a PV (Figure 2) facing the esophagus (the worst situation). Interlesion distance is $5.2 \mathrm{~mm}$ (such a distance is suggested to be less than $6 \mathrm{~mm}[4,13]$.

Voltage is tuned to achieve a power of $60 \mathrm{~W}$. Each application has the duration of $6 \mathrm{sec}$. Time between successive applications is $50 \mathrm{sec}$. We compute the LET time variation caused by the cumulated effect of repeated ablations in the presence of a multisensor esophageal thermal probe (ETP), with the central sensor placed at the point closest to the ablator. Specific reference is made to the FIAB 7-sensor Esotherm Multi probe. The choice of the $50 \mathrm{sec}$ time between successive application is tailored to the selected geometry. For our purposes a shorter time would have required a larger distance between the esophagus and the ablator in order to attain a similar result. The study of a correlation between such a time and the anatomical configuration giving comparable LET evolution is beyond the scope of the present paper.

We have considered three geometrical settings:

A. Left Atrium (LA) thickness 2.3mm, epicardial fat layer thickness $1 \mathrm{~mm}$, esophagus thickness $2.1 \mathrm{~mm}$, distance between esophagus and fat layer $0.5 \mathrm{~mm}$. PV cross section area $0.785 \mathrm{~cm}^{2}$, blood discharge through the $4 \mathrm{PVs}$ and through each cardiac chamber $5000 \mathrm{~cm}^{3} / \mathrm{min}$. These conditions correspond to frequent, not particularly critical - but border to critical - physiological data [14, $15]$.

B. Esophagus thickness $2.5 \mathrm{~mm}$, distance between esophagus and fat layer $1.5 \mathrm{~mm}$, other data as in (A). The overall sensor-ablator distance is thus increased by $1.2 \mathrm{~mm}$ compared to case $(\mathrm{A})$.

C. Anatomical configuration similar to (A), but with slightly thinner tissue layers: LA $2 \mathrm{~mm}$, esophagus $2 \mathrm{~mm}$, no fat layer. Cumulative reduction of sensor-ablator distance with respect to case $(A)$ is $1.4 \mathrm{~mm}$.

For (A) and (B) we have considered two sequences of applications:

i. Starting the sequence from Point 1, Figure 2.

ii. Starting the sequence from Point 4, Figure 2.

For (C) we limited ourselves to the sequence (I).

\section{Results}

The results shown here refer to the temperature computed on the ETP central sensor. Figure 3 compares the numerical simulations for the geometry (A) in the two sequences (I) (green line) and (II) (red line). Dots represent the temperatures taken every $2 \mathrm{sec}$. In the sequence (I) LET increases from $37^{\circ} \mathrm{C}$ to a maximum of $39.1^{\circ} \mathrm{C}$, in the sequence (II) it climbs to $39.7^{\circ} \mathrm{C}$. It means that if we start ablating from the remotest point, when we come to the closest one the heat already accumulated pushes LET to higher values. The LET increase rate in the first application of Sequence I is $\left(0.13^{\circ} \mathrm{C} / \mathrm{s}\right)$, a rather large value that

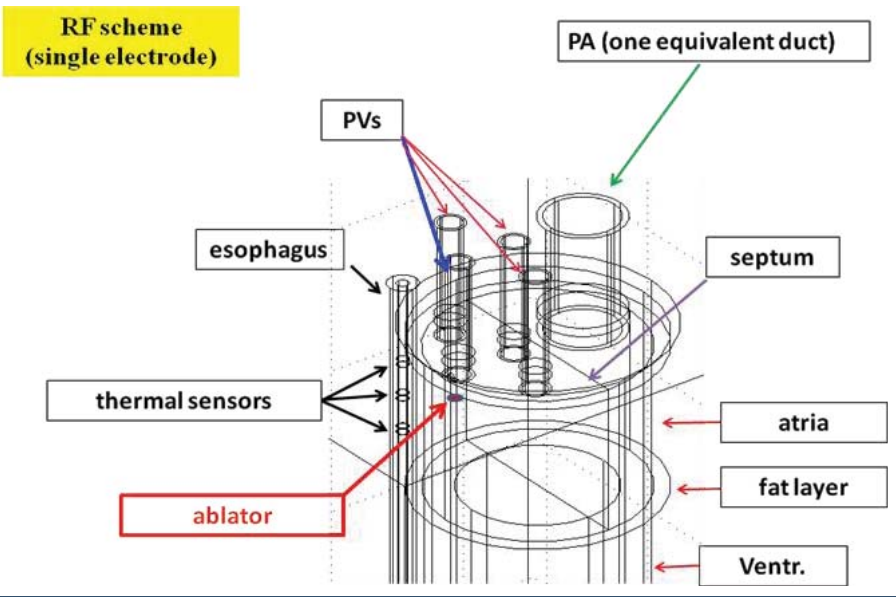

Figure 1: Simplified geometrical scheme used for numerical simulations (from [11])

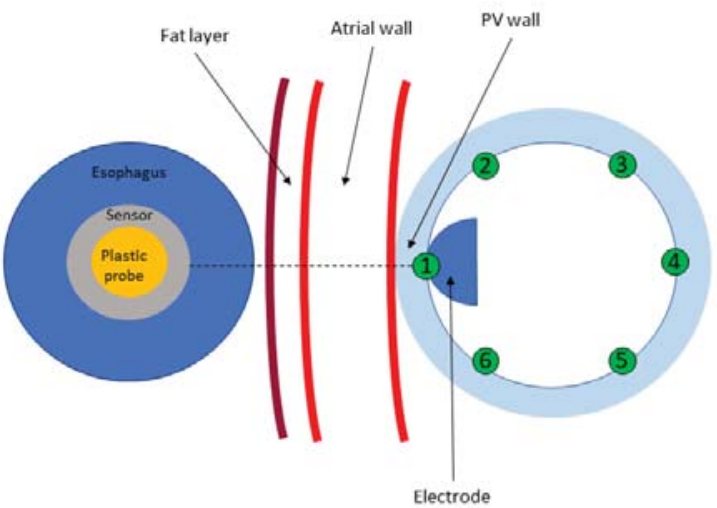

Figure 2: Sketch of the cross section through ablator. An irrigated ablator is applied at 6 evenly spaced points around the ostium of a PV facing the esophagus.

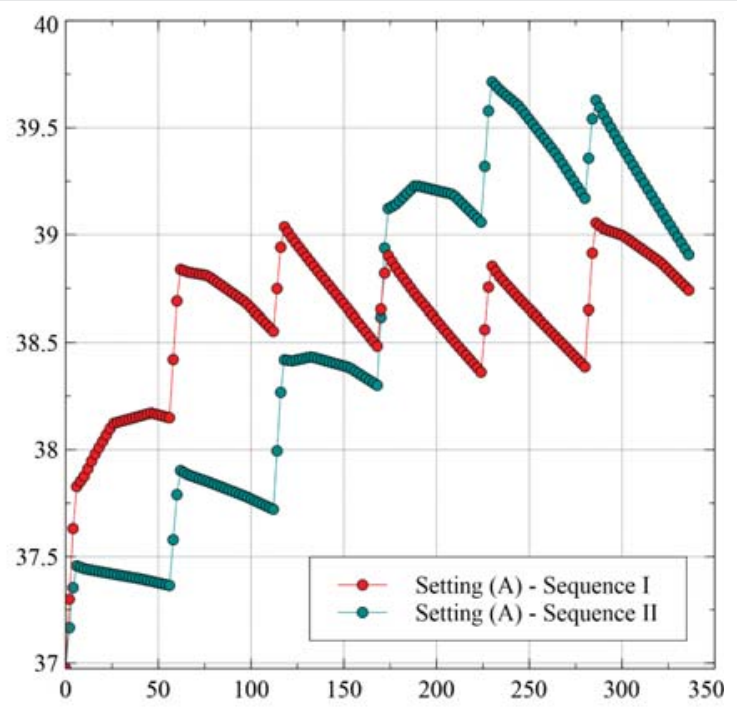

Figure 3: LET (central sensor) vs. time for geometry (A).Comparison of the numerical simulations for the geometry $(A)$ in the two sequences $(I)$ (red line) and (II) (green line). Dots represent the temperatures taken every 2 sec. Time scale in seconds. Ablation Sequence I produces eventually a lower max LET increase.

would be alarming in the traditional procedures, but is not here because of the short application time. Precisely the same rate is observed in the fourth ramp of Sequence II, which refers once 
more to Point 1 . Note that if the threshold LET value $39.5^{\circ}$ is adopted, as it frequently happens, Sequence II would trigger the alarm, while Sequence I would not.

Figure 4 illustrates the same comparison between Sequences I and II with reference to the geometrical Setting (B)

As expected, the slight increase of the sensor-ablator distance yields a moderate lowering of LET, the maximum difference between Sequence I and Sequence II being now reduced to $0.4^{\circ} \mathrm{C}$. Comparison between Setting (A) and Setting (B), confined to Sequence I, is shown in Figure 5.

The slight increase of the sensor-ablator distance results in lowering the maximum LET by approximately $0.5^{\circ} \mathrm{C}$. A similar difference is detected comparing the respective results for Sequence (II) (not shown here).

In contrast to the moderate changes when passing from (A) to (B), the results obtained for Setting (C), where the sensorablator distance is slightly reduced, show an impressive increase of the maximum LET. Since we have learned that Sequence (I) is the most convenient, we limited the computation to that case. Figure 6 illustrates the comparison between (A) and (C).

The maximum LET for Setting (C) is $45.8^{\circ} \mathrm{C}$, i.e. $6.7^{\circ} \mathrm{C}$ larger than in Case (A). This is a striking evidence that very small tissues compression can lead to definitely dangerous LET increase. LET stays above $40^{\circ} \mathrm{C}$ during almost all the procedure (for the considered PV) and beyond $42^{\circ} \mathrm{C}$ for about $2.5 \mathrm{~min}$, which is unacceptable. Equally impressive is the increase in the LET rate of change at the first application, passing from $0.13^{\circ} \mathrm{C} / \mathrm{s}$ (Setting A), to $0.3^{\circ} \mathrm{C} / \mathrm{s}$ (Setting C). The maximum LET increase detected by Barbhaiya, et al. [7]. in a clinical procedure was $5.8^{\circ} \mathrm{C}$, thus in a comparable range.

The remark about the LET rate of change stresses the importance of the ETP response time. Such a feature is not

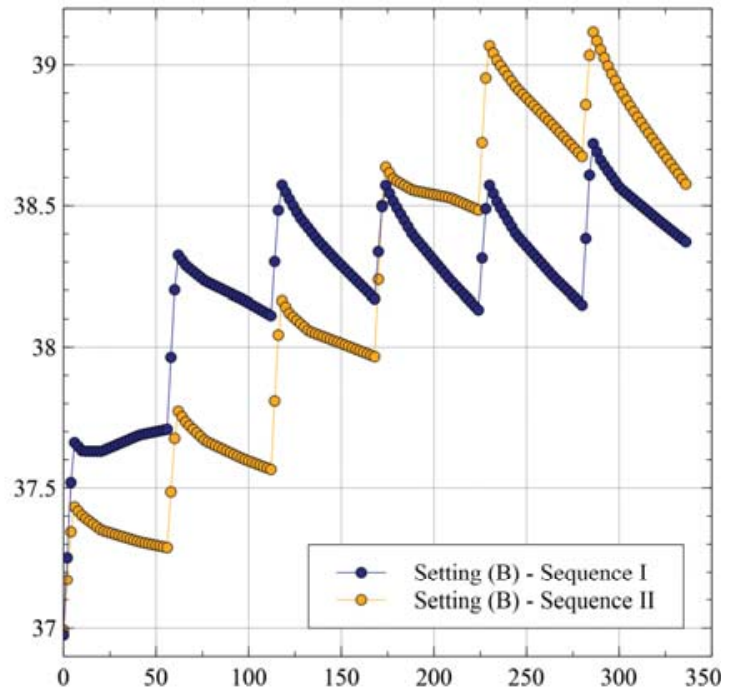

Figure 4: LET (central sensor) vs. time for geometry (B).Comparison of the numerical simulations for the geometry (B) in the two sequences (I) (blue line) and (II) (yellow line). Dots represent the temperatures taken every $2 \mathrm{sec}$. Time scale in seconds. Like for Setting A, also for Setting B it is preferable to adopt the ablation sequence I.

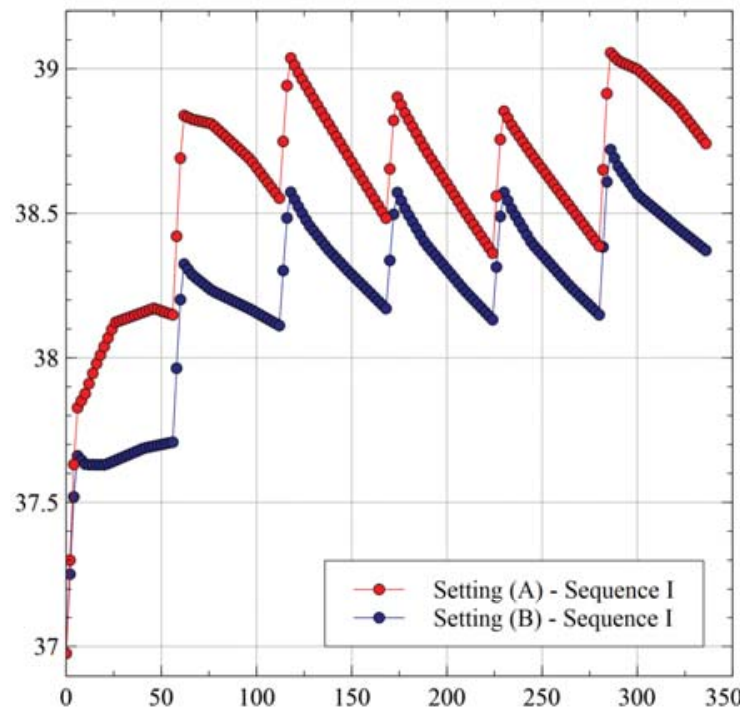

Figure 5: Comparison between Setting (A) and Setting (B), Sequence (I). The slight increase of the esophagus-ablator distance, passing from $A$ to $B$, results in a modest reduction of the LET increase and of the maximal LET increase rate.

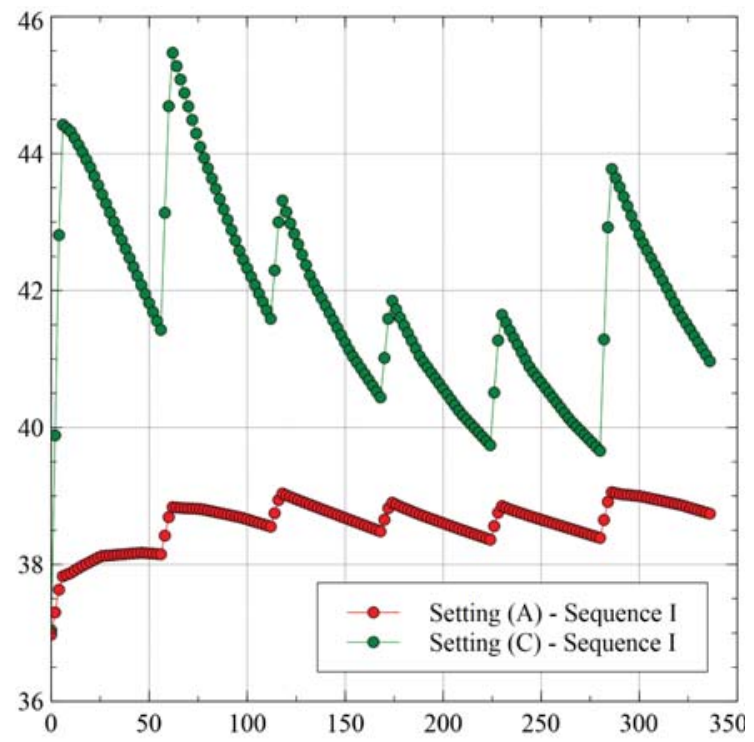

Figure 6: Comparison between Settings $(A)$ and $(C)$; Sequence (I). Setting $C$ differs from Setting A because of a small reduction of the thickness of the tissues interposed between the ETP and the ablator. The corresponding LET variation is dramatic, with a rather long permanence $(2.5 \mathrm{~min})$ beyond $42^{\circ} \mathrm{C}$. The max LET increase rate is also considerably larger.

enough emphasized in the literature concerning atrial ablation, but it is actually quite critical in the presence of rapidly changing temperatures (in such conditions response times exceeding 2 sec are likely to provide false information on LET evolution [16]).

\section{Discussions}

Our simulations show that HPSD ablation procedures are safe for most anatomical configurations. The Setting (A) represents a borderline configuration in the sense that a reduction of the esophagus-ablator distance as small as $1.4 \mathrm{~mm}$ leads to a dramatic LET increase (Setting C), while a comparatively small increase has little effect (Setting B). It must be kept in mind that contact force applied to the ablator may produce small tissue 
compression, thus potentially creating a dangerous situation. Such a possibility had been stressed in the literature [17]. It seems therefore crucial to monitor the esophageal temperature to ascertain whether a HPSD ablation is feasible or not, or in any case to calibrate the procedure to the patient's needs. LET variations depend also on the ablation sequence. Starting from the point in the PV which is closest to the esophagus leads eventually to a maximum LET that is lower than the one attained when starting from the farthest point (a useful indication for the clinician)

Owing to the possibility that LET increase rate can assume large values, it is very important that the response time of the ETP used is sufficiently small (less than 2s), otherwise the probe is likely to provide the operator with deceptively safe information.

\section{Conclusions}

We have computed the thermal field generated by an irrigated ablator during a HPSD ablation procedure. We established that

- With respect to a rather frequent anatomical configuration (Setting A), the procedure is safe.

- It is convenient to start the ablation sequence on a pulmonary vein from the point which is closest to the esophagus, otherwise LET will reach higher values.

- The selected reference configuration (Setting A) is border to critical: while a small increase of the sensorablator distance produces modest changes in LET, a comparably small reduction pushes LET to clearly unsafe values, since the esophagus is exposed to a steep thermal gradient.

- We get a clear indication that LET monitoring is of crucial importance and more specifically that it is necessary to use fast responding ETPs, since the LET variation speed can be very large.

\section{References}

1. Baher A, Kheirkhahan M, Rechenmacher SJ, Marashly Q, Kholmovski EG, et al. (2018) High-Power Radiofrequency Catheter Ablation of Atrial Fibrillation Using Late Gadolinium Enhancement MRI as a Novel Index of Esophageal Injury. JACC Clin Electrophysiol 4: 1583-1594. Link: https://bit.ly/2zy2u4g

2. Vassallo F, Cunha, Serpa E, Meigre LL, Carloni H, et al. (2019) Comparison of high power short duration (HPSD) ablation of atrial fibrillation using a contact force sensing catheter and conventional technique: Initial results. J Cardiovasc Electrophysiol 30: 1877-1883. Link: https://bit.ly/35XHrE5

3. Okamatsu H, Koyama J, Sakai Y, Negishi K, Hayashi K, et al. (2019) Highpower application is associated with shorter procedure time and higher rate of first-pass pulmonary vein isolation in ablation index-guided atrial fibrillation ablation. J Cardiovasc Electrophysiol 30: 2751-2758. Link: https://bit.ly/2T4BbWa

4. Berte B, Hilfiker G, Moccetti F, Schefer T, Weberndörfer V, et al. (2019) Pulmonary vein isolation using ablation index vs. CLOSE protocol with a surround flow ablation catheter. J Cardiovasc Electrophysiol 30: 2199-2204. Link: https://bit.ly/2T18jOB

5. Bunch TJ, May HT, Bair TL, Crandall BG, Cutler MJ, et al. (2020) Long-term outcomes after low power, slower movement versus high power, faster movement irrigated-tip catheter ablation for atrial fibrillation. Heart Rhythm 17: 184-189. Link: https://bit.ly/2WtdrwN

6. Reddy VY, Grimaldi M, De Potter T, Vijgen JM, Bulava A, et al. (2019) Pulmonary Vein Isolation with Very High Power, Short Duration, TemperatureControlled Lesions. The QDOT-FAST Trial. JACC: Clinical Electrophysiology 5: 778-786. Link: https://bit.ly/3cFsdWQ

7. Barbhaiya C R, Kogan EV, Jankelson L, Knottset RJ, et al. (2020) Esophagea Temperature Dynamics During High Power Short Duration Posterior Wall Ablation. Heart Rhythm 17: 721-727. Link: https://bit.ly/3bv9r3f

8. Berjano EJ, Hornero F (2004) Thermal-electrical modeling for epicardial atria radiofrequency ablation. IEEE Trans Biomed Engineering 51: 1348-1357. Link: https://bit.ly/2xYqxJb

9. Berjano EJ (2006) Theoretical modeling for radiofrequency ablation: state of the art and challenges for the future. Biomed Eng Online 5: 24. Link: https://bit.ly/2T3Qz57

10. Pérez JJ, D’Avila A, Aryana A, Berjano E (2015) Electrical and Thermal Effects of Esophageal Temperature Probes on Radiofrequency Catheter Ablation of Atrial Fibrillation: Results from a Computational Modeling Study. J Cardiovasc Electrophysiol 26: 556-564. Link: https://bit.ly/2WtH4Os

11. Fasano A, Anfuso L, Bozzi S, Pandozi C (2016) Safety and necessity of thermal esophageal probes during radiofrequency ablation for the treatment of atrial fibrillation. J Atrial Fibrillation 9: 1434. Link: https://bit.ly/3bv9QTj

12. Fasano A, Anfuso L, Arena G, Pandozi C (2017) Cryo-ablation for pulmonary veins isolation: importance of ETM. J Atrial Fibrillation 9: 1-7.

13. Dhillon G, Ahsan S, Honarbakhsh S, Lim W, Baca M, et al. (2019) A multicentered evaluation of ablation at higher power guided by ablation index: Establishing ablation targets for pulmonary vein isolation. J Cardiovasc Electrophysiol 30 357-365. Link: https://bit.ly/3dDHK9Z

14. Lemola K, Sneider M, Desjardins B, Case I, Han J, et al. (2004) Computed Tomographic Analysis of the Anatomy of the Left Atrium and the Esophagus Implications for Left Atrial Catheter Ablation. Circulation 110: 3655-3660. Link: https://bit.ly/3czLY2o

15. Tsao HM, Wu MH, Higa S, Lee KT, Tai CT, et al. (2005) Anatomic Relationship of the Esophagus and Left Atrium. Chest 28: 2581-2587. Link: https://bit.ly/3fNb7IA

16. Anfuso L, Corsi M, Fasano A (2018) Esophageal thermal probes: how fast should they be? M J Cardiol 3: 018. Link: https://bit.ly/2T1Qq1S

17. Black-Maier E, Pokorney SD, Barnett AS, Zeitler EP1, Sun AY, et al. (2017) Risk of atrioesophageal fistula formation with contact force-sensing catheters. Heart Rhythm 14: 1328-1333. Link: https://bit.ly/3buNhxU

Copyright: () 2020 Fasano A, et al. This is an open-access article distributed under the terms of the Creative Commons Attribution License, which permits unrestricted use, distribution, and reproduction in any medium, provided the original author and source are credited. 